\title{
IDENTIFICAÇÃO DE ESPÉCIES DE FORMIGAS CORTADEIRAS EM PLANTIOS DE Eucalyptus urograndis
}

\author{
Daniele Ukan ${ }^{1}$, Nilton José Sousa ${ }^{2}$, Páblo Geórgio de Souza ${ }^{3}$, Pedro Pacheco dos Santos Lima ${ }^{4}$ \\ ${ }^{1}$ Enga ${ }^{a}$ Florestal, Doutoranda em Ciências Florestais, UFPR, Curitiba, PR, Brasil - daniukan@ yahoo.com.br \\ ${ }^{2}$ Eng. Florestal, Dr., Depto. de Ciências Florestais, UFPR, Curitiba, PR, Brasil - nilton@ufpr.br \\ ${ }^{3}$ Eng. Florestal, Doutorando em Ciências Florestais, UFPR, Curitiba, PR, Brasil - pgsouza03@ gmail.com \\ ${ }^{4}$ Eng. Florestal, Dr., Depto. de Ciências Florestais, USP, São Paulo, SP, Brasil - ppacheco@usp.br
}

Recebido para publicação: 10/02/2009 - Aceito para publicação: 10/03/2010

\begin{abstract}
Resumo
Os objetivos deste trabalho foram: identificar as espécies de formigas cortadeiras que ocorrem em povoamentos de Eucalyptus urograndis localizados na região litorânea do estado de Santa Catarina; determinar o número médio de formigueiros por ha e a proporção de formigueiros por espécie de formiga cortadeira existente nos locais amostrados; relacionar as espécies existentes com danos provocados a mudas implantadas de E. urograndis. Os experimentos aqui relatados foram realizados na Fazenda Coqueiro e Fazenda Jacutinga, localizadas no litoral norte de SC, nos municípios de São João do Itaperiú e São Francisco do Sul, respectivamente. Os levantamentos realizados demonstram a presença das espécies Acromyrmex crassispinus (FOREL, 1909), Cyphomyrmex sp e uma espécie não Attini. Nas parcelas do Projeto Coqueiro encontram-se uma média de 143 formigueiros por hectare e na parcelas do Projeto Jacutinga encontram-se em média 77 formigueiros por hectare. A espécie A. crassispinus representa 33\% dos formigueiros encontrados, Cyphomyrmex sp. representa $40 \%$ dos formigueiros encontrados, enquanto que a espécie não Attini representa $27 \%$ dos formigueiros encontrados. A incidência de danos relacionados a formigas cortadeiras é pequena, estando associados à espécie A. crassispinus.

Palavras-chave: Formigas cortadeiras; identificação; Eucalyptus urograndis.
\end{abstract}

\begin{abstract}
Identification of leaf-cutting ants species in Eucalyptus urograndis plantations. The objectives of this work were: to identify the species of leaf-cutting ants that occur in Eucalyptus urograndis plantations located in the littoral region of the State of Santa Catarina; to determine the average number of formicaries per ha and the ratio of formicaries by species of existing leaf-cutting ants in the sampled areas; to correlate the existing species to the damages caused to the Eucalyptus plants. The experiments were carried out at the Coqueiro and Jacutinga farms, located in the municipalities of São João do Itaperiú and São Francisco do Sul, coastal region of Santa Catarina State, Brazil. The surveys indicated the presence of Acromyrmex crassispinus (FOREL, 1909), Cyphomyrmex sp and a non Attini species. An average of 143 and 77 formicaries per hectare were found in the sampled plots of Coqueiro and Jacutinga farms, respectively. The species A. crassispinus represented $33 \%$ of the observed formicaries; Cyphomyrmex sp. represented 40\%; whereas the non Attini species represented $27 \%$. The incidence of damages caused by leaf-cutting ants was small, mainly associated to the species $A$. crassispinus.

Keywords: Leaf-cutting ants; identification; Eucalyptus urograndis.
\end{abstract}

\section{INTRODUÇÃO}

Em florestas implantadas de Pinus sp. e de Eucalyptus sp., as formigas cortadeiras dos gêneros Atta (saúvas) e Acromyrmex (quenquéns) destacam-se como as principais pragas, especialmente nas fases de pré-corte e imediatamente após o plantio ou no início da condução de brotação. Por isso a preocupação com o controle desses insetos é constante.

Essa preocupação também é permanente em outros agroecossistemas, tornando o controle desses insetos uma exigência para qualquer empreendimento. Entre as alternativas para o seu controle, as iscas 
granuladas são amplamente utilizadas. Estima-se que no Brasil o consumo anual seja de aproximadamente 12.000 toneladas/ano de iscas tóxicas (FORTI, sem data).

Tradicionalmente, as empresas florestais consideram o controle de formigas cortadeiras como uma atividade imprescindível para o sucesso da implantação de povoamentos florestais, porém poucas empresas realizam essa atividade com base em parâmetros preestabelecidos, que determinem a melhor estratégia para a distribuição de iscas granuladas e as quantidades adequadas a serem distribuídas, não levando em consideração as espécies existentes e a quantidade de formigueiros na área. Com esse procedimento, desconsideram a citação de Lima (1991), que destaca que um dos pontos fundamentais para um bom planejamento de controle de formigas é a identificação das espécies que ocorrem em cada região.

Existe a hipótese de que as espécies de formigas cortadeiras encontradas em plantios florestais na região, segundo a literatura, não são espécies muito agressivas e causam poucos danos às mudas plantadas. Dessa forma, o planejamento do controle de formigas cortadeiras pode ser realizado em função das espécies encontradas, podendo ser reduzida a quantidade de produto químico utilizado para seu controle.

Partindo dessa premissa, este trabalho teve como objetivos: identificar as espécies de formigas cortadeiras que ocorrem em povoamentos de Eucalyptus urograndis localizados na região litorânea do estado de Santa Catarina; determinar o número médio de formigueiros por ha e a proporção de formigueiros por espécie de formiga cortadeira existente nos locais amostrados; relacionar as espécies existentes com danos provocados a mudas implantadas de $E$. urograndis.

\section{MATERIAIS E MÉTODOS}

Os experimentos deste trabalho foram realizados na Fazenda Coqueiro, localizada no município de São João do Itaperiú, SC, localizada a uma latitude de $26^{\circ} 37^{\prime} 04^{\prime \prime}$ sul e a uma longitude de $48^{\circ} 46^{\prime} 05^{\prime \prime}$ oeste, estando a uma altitude de 33 metros, e na na Fazenda Jacutinga, localizada no município de São Francisco do Sul, SC, localizada a uma latitude de $26^{\circ} 14^{\prime} 36^{\prime \prime}$ sul e a uma longitude de $48^{\circ} 38^{\prime} 17^{\prime \prime}$ oeste, a uma altitude de 9 metros (Figura 1). De acordo com a classificação de Köppen, o clima da região é caracterizado como Cfa: clima temperado úmido com verão quente.
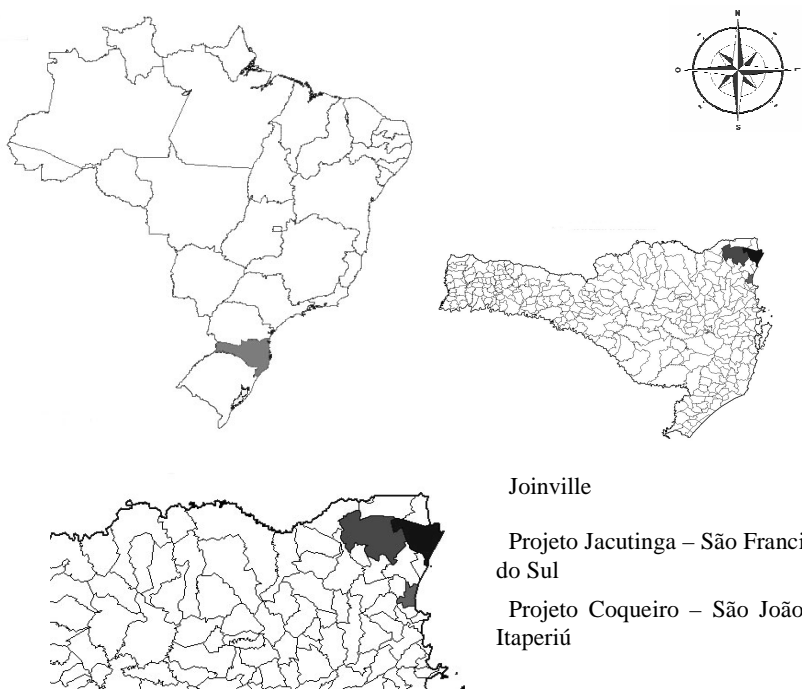

Joinville

Projeto Jacutinga - São Francisco do Sul

Projeto Coqueiro - São João do Itaperiú

Figura 1. Mapa da localização dos experimentos no estado de Santa Catarina. Adaptado de Siqueira (2008) e Centro de Informática e Automação do Estado de Santa Catarina - CIASC (2008). Escala dos mapas: (BRASIL 1:170.000.000) e (SC 1:10.000.000).

Figure 1. Map of the localization of the experiments in the State of Santa Catarina. Adapted of Siqueira (2008) and Center of Computer Science and Automation of the State of Santa Catarina CIASC (2008). Scale of the maps: (BRAZIL 1:170.000,000) e (SC 1:10.000,000). 
Foram instaladas 56 parcelas de $200 \mathrm{~m}^{2}$ na Fazenda Coqueiro e 56 parcelas de $100 \mathrm{~m}^{2}$ na Fazenda Jacutinga. A área total dos experimentos foi de aproximadamente $51.800 \mathrm{~m}^{2}(5,18 \mathrm{ha})$, ambas da empresa Comfloresta, do grupo Brascan Brasil.

As parcelas foram percorridas seguindo-se a linha de plantio, sendo contados e anotados todos os formigueiros visíveis. Destes, foram coletados exemplares para identificação das espécies presentes na região, que eram acondicionadas em vidros com álcool 70\%, para posteriormente serem montadas com alfinetes entomológicos no laboratório de Proteção Florestal do Curso de Engenharia Florestal da Universidade Federal do Paraná. Após a montagem, os exemplares coletados foram enviados para o Dr. Pedro Pacheco de Lima, Especialista da Universidade de São Paulo (USP), que procedeu a identificação dos mesmos. Também foi observada a ocorrência de danos às mudas de E. urograndis, sendo anotada a ocorrência ou não de dano.

\section{RESULTADOS E DISCUSSÃO}

Durante os levantamentos realizados, foram encontradas as espécies Acromyrmex crassispinus (FOREL, 1909), com os nomes populares de quenquém-de-cisco e quenquém, Cyphomyrmex sp. e uma espécie não Attini, porém muito parecida com Pheidole sp. (Tribo Pheidolini).

A espécie A. crassispinus representou 33\% dos formigueiros encontrados, corroborando as observações de Della Lúcia (1993), Gonçalves (1957) e Reis; Oliveira (2002), que citam que essa espécie tem ocorrência no estado de Santa Catarina, bem como nos estados do PR, RS, SP, RJ, MG e DF.

A espécie Cyphomyrmex sp. representou $40 \%$ dos formigueiros encontrados, enquanto que a espécie não Attini representou 27\% dos formigueiros encontrados.

No Projeto Coqueiro foram encontrados em média 143 formigueiros por hectare. No Projeto Jacutinga foram encontrados em média 77 formigueiros por hectare.

Os formigueiros observados ou contados em campo foram os que eram visíveis superficialmente. Em relação a A. crassispinus, essa característica é indicada por Gonçalves (1957), que cita que o ninho dessa espécie quase sempre é superficial, com uma só panela grande em parte situada em uma escavação rasa. Há, entretanto, ninhos inteiramente subterrâneos, que não são difíceis de encontrar em Santa Catarina e no Rio Grande do Sul.

Em relação aos ninhos de Cyphomyrmex, as amostragens deste trabalho comprovaram as observações de Weber (1972), que cita que essas formigas são usualmente pequenas e os ninhos, menores, sendo que os mais primitivos têm apenas uma entrada (olheiro) e podem ser formados num pedaço de tora quebrada ou embaixo de uma pedra coberta (não exposta ao sol). O fungo tem em média $40 \mathrm{~mm}$ de altura e $30 \mathrm{~mm}$ de comprimento, e seu substrato é formado de excrementos de insetos, pequenos fragmentos de madeira podre, carcaças de insetos, folhas e flores.

Quanto à outra espécie, também não foram obtidas informações quanto aos ninhos, visto que não foi possível a determinação da espécie. Porém é importante destacar que em campo não foi possível detectar visivelmente diferenças superficiais entre os formigueiros existentes na área experimental (Figura 2).

Essa constatação reforça a importância de identificar as espécies de formigas que ocorrem em uma área, pois, de acordo com Lima (1991), a identificação correta é o primeiro passo para a busca de informações que vão levar à utilização de medidas de controle eficientes.

Em relação aos danos provocados pelas formigas observadas na área experimental, verificou-se que, embora tenha sido encontrado um número considerável de formigueiros, poucas formigas foram encontradas cortando Eucalyptus urograndis. Provavelmente, isso se deve à presença de Cyphomyrmex sp. Della Lúcia (1993) cita que esse é um gênero primitivo, que cultiva fungos utilizando fezes de insetos, matéria orgânica vegetal morta e cadáveres de insetos em geral como substrato. Esse comentário de Della Lúcia induz a uma percepção de que essa espécie não corta material vegetal, consequentemente, mudas de eucaliptos.

Quanto à espécie A. crassispinus, foram observados poucos ataques às mudas de E. urograndis. Ocorreram situações como a destacada na figura 3 , em que o olheiro do formigueiro situava-se na base da muda e esta não continha nenhum tipo de dano causado pelas cortadeiras. Esse comportamento pode estar associado ao período de atividade dessa espécie, pois, segundo Reis; Oliveira (2002), ele é acentuado entre os meses de dezembro a abril, sendo críticos os meses de fevereiro, março e abril. Como os experimentos foram realizados no período entre maio e julho, talvez esse seja o motivo que justifica a baixa atividade de forrageamento observada em campo. 


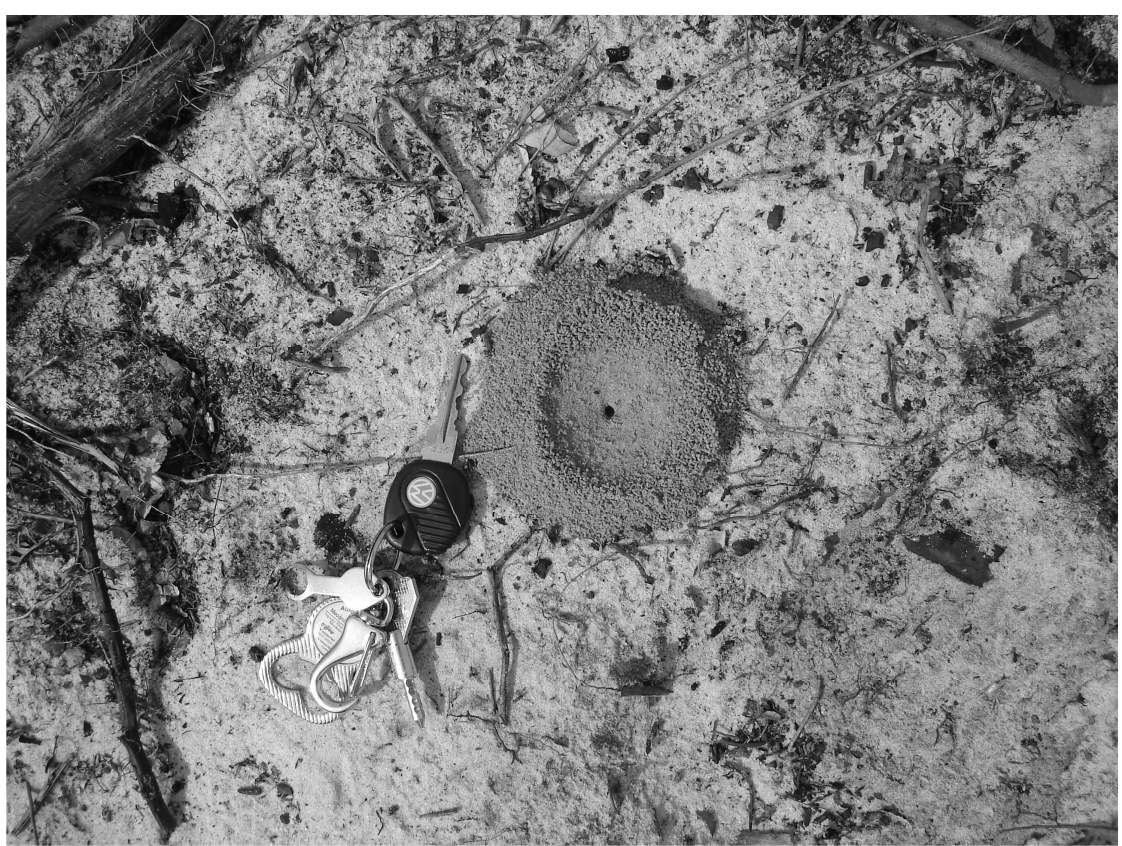

Figura 2. Amostra de um formigueiro, espécie não identificada, encontrado no projeto Coqueiro. São João do Itaperiú, SC.

Figure 2. Sample of creeps, species not identified, found in the project Coqueiro. São João do Itaperiú, SC.

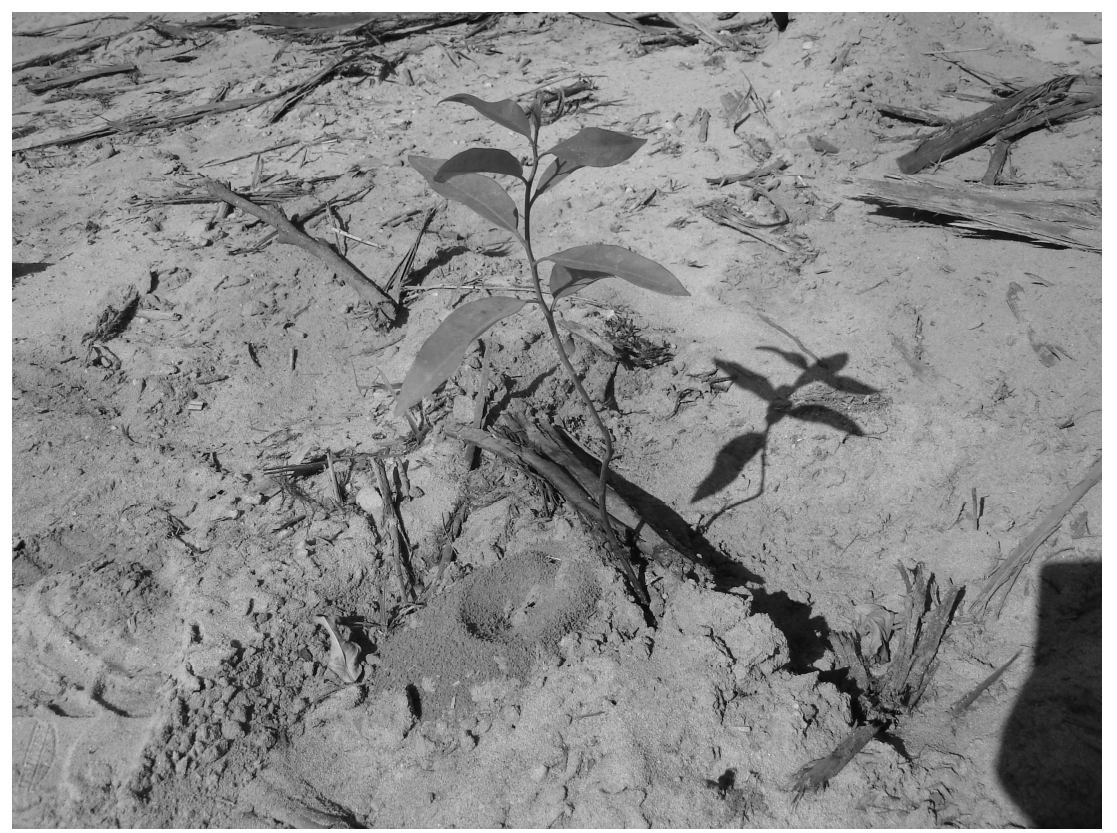

Figura 3. Formigueiro, espécie não identificada, próximo a muda de Eucalyptus urograndis, porém sem causar dano. São João do Itaperiú, SC.

Figure 3. Creeps, species not identified, next the plant to Eucalyptus urograndis, however, without damage. São João do Itaperiú, SC. 


\section{CONCLUSÕES}

Com base nos resultados obtidos neste trabalho, pode-se concluir que:

- Os levantamentos realizados demonstram a presença das espécies Acromyrmex crassispinus (FOREL, 1909), Cyphomyrmex sp. e uma espécie não Attini;

- Nas parcelas do Projeto Coqueiro encontra-se uma média de 143 formigueiros por hectare, e na parcelas do Projeto Jacutinga encontram-se em média 77 formigueiros por hectare;

- A espécie A. crassispinus representa 33\% dos formigueiros encontrados, Cyphomyrmex sp. representa $40 \%$ dos formigueiros encontrados, enquanto que a espécie não Attini representa $27 \%$ dos formigueiros encontrados;

- A incidência de danos relacionados a formigas cortadeiras é pequena, estando associada à espécie $A$. crassispinus.

- A hipótese levantada no trabalho foi aceita, pois os danos observados nas mudas de Eucalyptus urograndis, causados pelas espécies de formigas cortadeiras encontradas no plantio, foi baixo. Sendo assim, o planejamento do controle dessa praga deve ser realizado com a finalidade de reduzir a quantidade de produto químico utilizado, evitando desperdícios com o produto e de mão de obra, isto é, reduzindo custos de controle e beneficiando o meio ambiente, pois este receberá uma menor dose do produto químico.

\section{AGRADECIMENTOS}

À empresa COMFLORESTA, pela estrutura financeira e a pela cessão das áreas para a realização dos experimentos. Ao Professor Dr. Pedro Pacheco dos Santos Lima (USP), que procedeu a identificação do material biológico. À Fundação de Pesquisas Florestais do Paraná (FUPEF), pela estrutura administrativa que viabilizou a realização deste trabalho.

\section{REFERÊNCIAS}

CENTRO DE INFORMÁTICA E AUTOMAÇÃO DO ESTADO DE SANTA CATARINA. Mapa interativo de Santa Catarina. <www.mapainterativo.ciasc.gov.br>. Acesso em 07/01/2008.

DELLA LÚCIA, T. M. C. As formigas cortadeiras. Editado por Terezinha M. C. Della Lúcia. Minas Gerais, Viçosa, 1993. 262 p.

FORTI, L. C. Formigas cortadeiras. <www.uesb.br/entomologia/cort.html>. Acesso em 14/01/2008.

GONÇALVES, C. R. O Gênero Acromyrmex no Brasil. Tese para o concurso da cadeira de Entomologia e Parasitologia Agrícola da Escola Nacional de Agronomia. Rio de Janeiro, 1957, 81 p.

LIMA, P. P. S. Formigas cortadeiras (Hymenoptera, Formicidae) com ênfase nas culturas de pinus e eucaliptos. Dissertação de mestrado. São Paulo. Escola Superior de Agricultura Luiz de Queiroz. 86 p. Piracicaba, 1991.

REIS, F. W.; OLIVEIRA, S. Atividade externa, carregamento de isca granulada e controle de Acromyrmex crassispinus em floresta de Pinus taeda. Comunicado Técnico ${ }^{\circ}$ 78, Colombo, PR, 2002.

SIQUEIRA, S. Divisão estadual do Brasil. <www.pmf.sc.gov.br>. Acesso em 07/01/2008.

WEBER, N. A. Gardening ants: the Attines. Americam Philosofical Society. 1972. 166 p. 
FLORESTA, Curitiba, PR, v. 40, n. 4, p. 819-824, out./dez. 2010.

Ukan, D. et al. 